\title{
Comparison of Bayesian objective procedures for variable selection in linear regression
}

\author{
Elías Moreno • F. Javier Girón
}

Received: 18 February 2008 / Published online: 5 March 2008

(C) Sociedad de Estadística e Investigación Operativa 2008

\section{Erratum to: Test}

\section{DOI 10.1007/s11749-006-0039-1}

The original version of this article unfortunately contained flaws in Lemma 3.1 and Theorem 3.2. The corrected Lemma 3.1 and Theorem 3.2 are given below:

Lemma 3.1 The lower bound of the Bayes factor $(3.5), \inf _{\mathcal{B} \in[0,1]} B_{1 i}^{*}(n, \mathcal{B})=$ $B_{1 i}^{*}(n, 0)$ is given by

$$
\begin{aligned}
& \left(\frac{1}{\pi}\left(\frac{n}{i+1}\right)^{(n-i) / 2} B\left(\frac{1+i-n}{2}, \frac{1}{2}\right)\right. \\
& \left.\quad \times{ }_{2} F_{1}\left(\frac{i-n}{2}, \frac{1+i-n}{2}, \frac{2+i-n}{2} ;-\frac{i+1}{n}\right)\right)^{-1},
\end{aligned}
$$

where ${ }_{2} F_{1}(a, b, c ; z)$ denotes the hypergeometric function.

Further, for all $i \geq n$, the inf is an increasing function of $i$.

The online version of the original article can be found under http://dx.doi.org/10.1007/s11749-006-0039-1.

E. Moreno

Departamento de Estadística, Universidad de Granada, Granada, Spain

F.J. Girón ( $\varangle)$

Departamento de Estadística, Universidad de Málaga, Malaga, Spain

e-mail: fj_giron@uma.es 
Proof As the Bayes factor is an increasing function of the statistic $\mathcal{B}$, the minimum is attained at $\mathcal{B}=0$. For $\mathcal{B}=0$, the Bayes factor (3.5) reduces to the expression

$$
B_{1 i}^{*}(n, 0)=\left(\frac{2}{\pi} \int_{0}^{\pi / 2}\left(1+\frac{n}{(i+1) \sin ^{2} \varphi}\right)^{(n-i) / 2} d \varphi\right)^{-1} .
$$

This inf becomes 0 for $i<n$ as the integral diverges to infinity, and is equal to (3.8) for $i \geq n$. Note that for $i=n$, the inf equals 1. The proof that (3.8) is increasing in $i$, for $i \geq n$, is somewhat cumbersome as the proof uses some specific properties of the hypergeometric function and hence it is omitted.

Theorem 3.2 For any model $M_{i}$ with $i$ regressors such that $i \geq n$, the posterior probability $P^{*}\left(M_{i} \mid n, \mathcal{B}_{1 i}^{*}\right)$ is a decreasing function of $i$ that attains its maximum at $i=n$; the maximum of this probability being $1 / 2$.

Proof As $i \geq n$, the value of the $R_{i}^{2}$ statistic is equal to 1 and consequently $\mathcal{B}_{1 i}^{*}$ is equal to 0 ; therefore, from Lemma 1 and the fact that the posterior probability is a monotonic decreasing function of the Bayes factor, the theorem follows suit.

Acknowledgements The authors want to thank Guido Consonni and Piero Veronese for pointing out a flaw in the statement and proof of Lemma 3.1 and Theorem 3.2. 